\title{
Supplier's commodity pricing model based on non-cooperative game
}

\author{
theory \\ YANG Mao \\ Department of Economic-Managerial, Lanzhou institute of technology, 730050, China \\ Email: 21741646@qq.com
}

\begin{abstract}
Keywords: E-commerce, Pricing Strategy, Supplier, Competition, Doctor, Cooperation, Coordination
\end{abstract}

\begin{abstract}
With the application of conditional free shipping policy becoming more and more extensive, there are more and more research theories for it. How to confirm free shipping conditions and shipping cost is becoming an important problem faced by e-commerce enterprises. Make example analysis for a series of solutions generated based on algorithm design and C\# program design solving model and attain some conclusions: when the product price is within the psychological expectation range of consumers for this product, free shipping threshold is set at the boundary of all product price portfolios and situation of retailer profit jump will happen, in addition, the optimal free shipping threshold should be set around the mean value where the consumer expectation distributes. Of course, the pricing strategy of the optimal delivery service in enterprise is also affected by various factors; its forms are complicated and depend on the specific value of parameters.
\end{abstract}

\section{Introduction}

After entering into the 21st century, the amazing development speed of e-commerce starts to show its superiority. Monitoring data in China e-commerce research center has shown that in 2007, the transaction amount of online retail market is 52 billion Yuan and till 2011, the number breaks 800 billion Yuan and reaches 801.9 billion Yuan [1]. Based on the prediction of current develop trend, online sales market is still in the rapid development stage. Facing up with such a big and rapidly increasing market, both mature online retailers and emerging online retailers are with great growth potentiality. How to attract customers to buy and increase the market share of enterprise is becoming a problem faced by e-commerce enterprises. "Free shipping” has been adopted by more and more online retailers as one unique promotion method. Retailers motivate consumers to increase purchasing amount by setting a threshold for free shipping, combine several orders into one and decrease frequent small amount order. One extremely big free shipping threshold may not have the function of motivating consumers, but one extremely small free shipping threshold may decrease the profit of retailers for bearing more shipping cost. How to make free shipping rules and design logistics pricing strategy to maximize the profit of retailers and market share has become a common issue for practical circle and academic circle. 


\section{Model building}

\section{Problem description}

These two products can describe the purchasing decisions of consumers when having several products to choose. This paper sets two kinds of products sold by retailers. Table 1 is the parameter and variable for model.

Table 1 Symbol and meaning

\begin{tabular}{ll|ll}
\hline Symbol & Meaning & Symbol & Meaning \\
\hline$p_{j}$ & Price of product $j$ & $m_{j}$ & Margin profit rate of product $j$ (profit/cost) \\
$r$ & Logistics cost of each order for retailer & $q_{i j}$ & Quantity of product $j$ purchased by No. $i$ consumer \\
$i$ & Consumer $i$ (total number of consumers is & $\theta_{i j}$ & Psychological expectation of consumer $i$ for product $j$, \\
& $N)$ & & \multicolumn{2}{|c}{ Free shipping threshold } \\
$A_{i}$ & Purchasing mount of consuming $i$ & $T$ & \\
\hline
\end{tabular}

To study the reaction degree of consumers for retailer variables (product price, free shipping threshold, cost of order processing, cost), following assumptions have been made for model:

1) Consumers are rational, which is that consumers consume based on the principle of maximizing utility under the condition of within their consumption ability.

2) Based on the square root utility function adopted by scholars in literature review, assume the relationship of consumer utility and consumed product quantity as well as the psychological expectation of consumers for products is as following:

$u_{i j}=\theta_{i j} \sqrt{q_{i j}}(1)$

3) Shipping cost function charged by enterprise is:

$$
S\left(q_{j}\right)= \begin{cases}s & \text { if } \sum_{j} p_{j} q_{i j}<T \\ 0 & \text { if } \sum_{j} p_{j} q_{i j} \geq T\end{cases}
$$

Solution for consumer behavior model

Based on free shipping rules made by retailers, when the purchasing amount of consumers is smaller than $T$, consumer has to pay $s$ for delivery; when the purchasing amount of consumer is bigger than $T$, consumer can enjoy the free shipping. Based on the assumption that all the consumers are rational, the following surplus function can be established for each consumer:

$$
G(q 1, q 2)= \begin{cases}\sum_{j=1}^{2}\left(\theta_{j} \sqrt{q_{j}}-p_{j} q_{j}\right)-s & \text { if } \sum_{j=1}^{2} p_{j} q_{j}<T \\ \sum_{j=1}^{2}\left(\theta_{j} \sqrt{q_{j}}-p_{j} q_{j}\right) & \text { if } \sum_{j=1}^{2} p_{j} q_{j} \geq T\end{cases}
$$

Relationship between consumer purchasing amount and $\theta, s, T$ can be attained through maximizing this consumer surplus function. One set of algorithm has been designed in this paper to solve the optimization problem and the main thinking of algorithm is as following:

A. First, solve this integer constraint relaxation of integer programming problem to get one local optimal solution: $\left(q_{1}, q_{2}\right)=\left[\left(\frac{\theta_{1}}{2 p_{1}}\right)^{2},\left(\frac{\theta_{2}}{2 p_{2}}\right)^{2}\right]$.

B. Round up and down respectively for local optimal solution $\left(q_{1}, q_{2}\right)$ of linear program, set $q_{1}^{C}=\left\lceil q_{1}\right\rceil, \quad q_{1}^{F}=\left\lfloor q_{1}\right\rfloor, \quad q_{2}^{C}=\left\lceil q_{2}\right\rceil, \quad q_{2}^{F}=\left\lfloor q_{2}\right\rfloor$

C. When $p_{1} q_{1}^{c}+p_{2} q_{2}^{c} \geq T$, (consumer reaches the condition for free shipping at this moment), compare the size of $G\left(q_{1}^{C}, q_{2}{ }^{C}\right), G\left(q_{1}{ }^{C}, q_{2}{ }^{F}\right), G\left(q_{1}^{F}, q_{2}{ }^{C}\right), G\left(q_{1}{ }^{F}, q_{2}{ }^{F}\right)$ and attain the optimal purchasing amount of these two products as $\left(q_{1}, q_{2}\right)=\left\{\vec{q}, \max \left(G\left(q_{1}^{C}, q_{2}{ }^{C}\right), G\left(q_{1}^{C}, q_{2}{ }^{F}\right), G\left(q_{1}{ }^{\mathrm{F}}, q_{2}{ }^{C}\right), G\left(q_{1}{ }^{\mathrm{F}}, q_{2}{ }^{\mathrm{F}}\right)\right)\right\}$ 


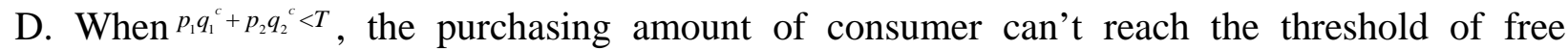
shipping and consumer will have two choices: keep the existing purchasing and pays shipping cost; increase purchasing amount to make it reach the threshold of free shipping. At this moment, retailers have more than one product and consumer can increase the purchasing amount of one product or two products to research the purchasing amount for free shipping; in addition, the selection of consumer for increasing purchasing amount is made based on the increase of consumer surplus. The following process is to judge whether the consumer will increase purchasing amount and how much is the increased purchasing amount. Set $\left(q_{1}^{*}, q_{2}^{*}\right)=\left\{\hat{q}, \max \left(G\left(q_{1}^{C}, q_{2}^{C}\right), G\left(q_{1}^{C}, q_{2}{ }^{F}\right), G\left(q_{1}^{F}, q_{2}^{C}\right), G\left(q_{1}^{F}, q_{2}{ }^{F}\right)\right)\right\}$,

1 select natural number $a=0$, judge if Ceiling $\left[\left(T-p_{1}\left(q_{1}{ }^{\mathrm{F}}+\mathrm{a}\right)\right) / \mathrm{p}_{2}\right]$ is bigger than 0 , if yes, go the second step, if no, go to the fifth step;

2 set $\mathrm{Q}_{1}=\mathrm{q}_{1}{ }^{\mathrm{F}}+\mathrm{a}, \mathrm{Q}_{2}=$ Ceiling $\left[\left(T-p_{1}\left(\mathrm{q}_{1}{ }^{\mathrm{F}}+\mathrm{a}\right)\right) / \mathrm{p}_{2}\right]$, if $G\left(\mathrm{Q}_{1}, \mathrm{Q}_{2}\right)>G\left(q_{1}^{*}, q_{2}{ }^{*}\right)$ and then turn to the third step or else turn to step 4.

3 set $q_{1}^{*}=Q 1, \quad q_{2}^{*}=Q_{2}$

$4 \mathrm{a}=\mathrm{a}+1$, return to the first step

5 judge if $G\left(q_{1}^{*}, q_{2}^{*}\right)$ is bigger than 0 , is the optimal output purchasing amount $\left(q_{1}^{*}, q_{2}^{*}\right)$, or else $\left(q_{1}^{*}, q_{2}^{*}\right)$, stop searching and output $\left(q_{1}^{*}, q_{2}^{*}\right)$.

Based on above algorithm, the purchasing decision of consumer under given conditions has been attained through C\# program. In reality, the distribution of consumer's expectation for one product is "concentrated in middle and scattered at two ends", which is approximate normal distribution. This paper has processed the values of psychological expectation value $\theta_{i 1}$ and $\theta_{i 2}$, which is to set two step lengths and simulate $\theta_{i 1}$ and $\theta_{i 2}$ into approximate normal distribution through changing step length. Picture 1 is the interface for solving consumer behavior model.

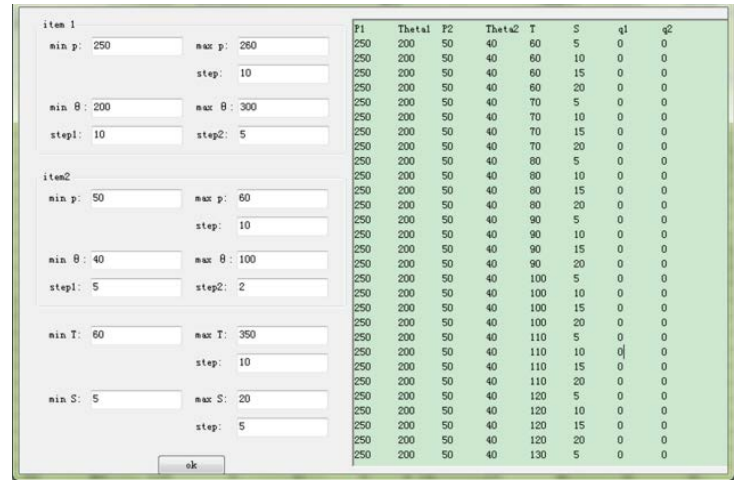

Figure 1 Interface for solving consumer behavior model

Solving for retailer profit model

Based on above solving program for consumer behavior model, the setting of parameters is as shown in picture 2 (the parameter selection here is based on the analysis of actual situation. Order processing cost of current mainstream e-commerce has been attained through investigation, at about 15-25 RMB/order and here select order processing cost at 15RMB/order; product marginal profit=profit/cost, the marginal profit of general living things is about $0.1-0.3$, the marginal profit of 3C products is about $0.3-0.5$ and here select marginal profit value of 0.15 ; shipping cost selects 5 , 10, 15, 20 from 5-20 which can cover the shipping cost charged by most of current e-commerce enterprises.), 222,720 consumer purchasing records have been attained. This paper has processed 
222,720 records and set up the relationship between retailer profit and consumer purchasing decision. Table 2 indicates the processed data (note: here only intercept partial data) and processing logic is as following:

Consumer purchasing amount $\mathrm{A}=$ product price $\mathrm{p} 1$ *consumer purchasing quality $\mathrm{q} 1+$ product price p2* consumer purchasing amount q2

Shipping cost paid by consumer $\mathrm{si}=$ if(consumer purchasing amount $<>0$, if (consumer purchasing amount $<$ threshold of free shipping $\mathrm{T}$, shipping cost $\mathrm{s}, 0), 0$ )

Retailer profit $\Pi=$ if (consumer purchasing amount $<>0$, consumer purchasing amount*product marginal profit + cost paid by consumer-order processing cost, 0 )

Table 2 Relationship between retailer profit and each variable

\begin{tabular}{lllllll}
\hline $\boldsymbol{P}_{1}$ & $\mathbf{5 0}$ & $\mathbf{5 0}$ & $\mathbf{5 0}$ & $\mathbf{5 0}$ & $\mathbf{5 0}$ & $\mathbf{5 0}$ \\
$\boldsymbol{\theta}_{\mathbf{1}}$ & 50 & 50 & 100 & 100 & 100 & 100 \\
$\boldsymbol{P}_{2}$ & 200 & 200 & 200 & 200 & 200 & 200 \\
$\boldsymbol{\theta}_{2}$ & 190 & 205 & 190 & 235 & 235 & 255 \\
$\boldsymbol{T}$ & 50 & 150 & 280 & 260 & 270 & 390 \\
$\boldsymbol{S}$ & 5 & 5 & 20 & 20 & 5 & 15 \\
$\boldsymbol{q}_{\mathbf{1}}$ & 1 & 1 & 2 & 2 & 1 & 1 \\
$\boldsymbol{q}_{2}$ & 0 & 1 & 1 & 1 & 1 & 1 \\
$\boldsymbol{m}$ & 0.15 & 0.15 & 0.15 & 0.15 & 0.15 & 0.15 \\
$\boldsymbol{r}$ & 15 & 15 & 15 & 15 & 15 & 15 \\
$\boldsymbol{A}$ & 50 & 250 & 300 & 300 & 250 & 250 \\
$\boldsymbol{s}_{\boldsymbol{i}}$ & 0 & 0 & 0 & 0 & 5 & 15 \\
$\Pi$ & -7.5 & 22.5 & 30 & 30 & 27.5 & 37.5 \\
\hline
\end{tabular}

\section{Conclusions}

This paper has taken the shipping cost strategy of current B2C enterprises as research object and established one two-stage model to solve the pricing strategy for delivery service of online retailer enterprises with the idea of game theory. In the paper, this problem has been abstracted into one two-stage game model: first, consumer confirms purchasing decision based on the principle of maximum utility and then consumer sets logistics pricing strategy based on the principle of profit maximization on the basis of considering about the purchasing decision of consumer. This paper has found out the retailer profit will jump when the threshold of free shipping is set at the boundary of product price portfolio through algorithm design and example analysis. As an exploratory research, some shortages and limitations exist in this paper, for example in reality, the total weight, total volume of goods as well as the geological location of clients will affect the shipping cost strategy design for retailer.

\section{Reference}

[1]Yishuang Geng, Kaveh Pahlavan, On the accuracy of rf and image processing based hybrid localization for wireless capsule endoscopy, IEEE Wireless Communications and Networking Conference (WCNC), Mar.2015.

[2]Jie He, Yishuang Geng, Fei Liu, Cheng Xu, CC-KF: Enhanced TOA Performance in Multipath and NLOS Indoor Extreme Environment, IEEE Sensor Journal, 14(11), 3766-3774, Nov. 2014.

[3]Lin Y, Yang J, Lv Z, et al. A Self-Assessment Stereo Capture Model Applicable to the Internet of Things[J]. Sensors, 2015, 15(8): 20925-20944. 
[4]Yang J, He S, Lin Y, et al. Multimedia cloud transmission and storage system based on internet of things[J]. Multimedia Tools and Applications, 2015: 1-16. 